\title{
APORTACIÓN A LOS CONCEPTOS DE TURISMO MARINERO/PESQUERO Y PESCA-TURISMO
}

\author{
Daniel Moreno Muñoz.
}

Universidad de Murcia

\section{RESUMEN}

En los últimos años, los lugares costeros con una fuerte tradición pesquera intentan fomentar una nueva modalidad turística, el denominado turismo marinero/pesquero. Sin embargo, aún no se ha definido de forma unánime el concepto, ya que las distintas Leyes de Pesca presentan para esta actividad una diversidad de significados. En esta investigación se muestran los resultados del análisis de la legislación existente en esta materia, así como de la literatura científica, y se aporta una nueva definición de turismo/pesquero y de pescaturismo, con el fin de aclarar ambos términos.

Palabras clave: Turismo marinero/pesquero; pesca artesanal; desarrollo sostenible.

\section{Contribution to the concepts of fishing tourism and pesca-tourism}

\section{ABSTRACT}

In recent years, coastal places with a strong fishing tradition are trying to promote a new tourism modality, the so-called marine / fishing tourism. However, the concept has not yet been unanimously defined, since the different Fishing Laws present a variety of clarifications for this activity. This research shows the results of the analysis of existing legislation in this area, as well as the scientific literature, and provides a new definition of tourism / fishing and fishing-tourism, in order to clarify both terms.

Keywords: Fishing Tourism; artisanal fisheries; sustainable development.

Fecha de recepción: 16 de enero de 2018.

Fecha de aceptación: 9 de junio de 2018.

Grupo de Investigación “Dinámicas Territoriales. Análisis y Ordenación”. Universidad de Murcia. E-mail: daniel.moreno1@um.es 


\section{INTRODUCCIÓN}

El turismo marinero/pesquero es una actividad de reciente implantación que está siendo fomentada en distintas zonas costeras del mundo (Pardellas y Espejo, 2013). De acuerdo con Herrera-Racionero et al. (2018), este hecho viene motivado por la crisis socio-ambiental que está viviendo la pesca tradicional, con importantes costes económicos y culturales, por lo que se está estimulando la búsqueda de alternativas que concilien la supervivencia de la pesca artesanal, con gran arraigo en las poblaciones litorales, con la necesaria sostenibilidad ambiental.

En esta situación de crisis del sector pesquero y de búsqueda de nuevas singularidades competitivas para atraer a un mayor número de turistas, surge el pensamiento sobre si una actividad novedosa como el turismo marinero/pesquero puede convertirse en un complemento económico para los pescadores, y que genere un beneficio socioeconómico en el territorio inmediato (Moreno, 2016).

La pesca constituye, por sí misma, una actividad vivencial, en contacto con un ecosistema como es el marino, lleno de sugerencias y matices; una experiencia que despierta gran interés para el visitante: sus recursos naturales, pesqueros y culturales (faro, puertos, lonjas de pescado, los sistemas y artes tradicionales de pesca, el contacto con las gentes del mar y su vocabulario propio, un conjunto de intensas manifestaciones festivas asociadas con el mar como marco inspirador y como escenario donde llevarse a cabo, sus museos y centros de interpretación, gastronomía, monumentos y artesanía).

Por tanto, el pilar básico sobre el que se sustenta esta modalidad turística es el patrimonio cultural pesquero, tangible e intangible, suspicaz de ser aprovechado como un recurso turístico (Moreno, 2018). Es decir, tal y como afirma Capel (2014), es una oportunidad para poner en valor elementos como los espacios y la arquitectura portuaria histórica, equipamientos pesqueros, arsenales históricos o astilleros navales; en definitiva, todo el poblamiento y los paisajes del litoral. No obstante, también deben ser considerados otros aspectos ligados al mar como las tradiciones y saberes locales, la gastronomía o las festividades religiosas (Figura 1) (Martínez y Moreno, 2013).

A nivel internacional se han constatado iniciativas en América, Asia y Europa. Su desarrollo no es homogéneo, siendo los países ribereños del Mar Mediterráneo donde más se ha impulsado esta modalidad turística. Conviene, por tanto, citar algunos de ellos. En Brasil, concretamente en Carnaubal (Piauí), los pescadores artesanales están comenzando a transportar a los turistas a contemplar la faena pesquera con el fin de poner en valor sus oficios y conseguir nuevas rentas (Vasconcelos et al., 2014).

En Asia, los pescadores coreanos transportan a visitantes a los caladeros para que contemplen la actividad pesquera. Incluso les dan alojamiento en sus viviendas, y con ello consiguen recursos económicos. A esta situación les lleva la disminución de los recursos pesqueros, y, por tanto, la caída de sus ingresos (Cheong, 2003). En Taiwan, los marineros, debido a la disminución de ingresos y el aumento desmesurado del turismo de masas, son partícipes de la actividad pesca-turismo, mostrando una satisfacción general con ésta y con los operadores turísticos (Chen y Chang, 2017).

En Europa destacan iniciativas como la de Istria (Eslovenia), Sicilia (Italia) o la Região Centro Norte de Portugal. En el primer caso, debido al incremento del turismo, la pobla- 


\section{Figura 1 \\ PROCESIÓN MARÍTIMA EN HONOR A LA VIRGEN DEL CARMEN. MAZARRÓN. ESPAÑA}

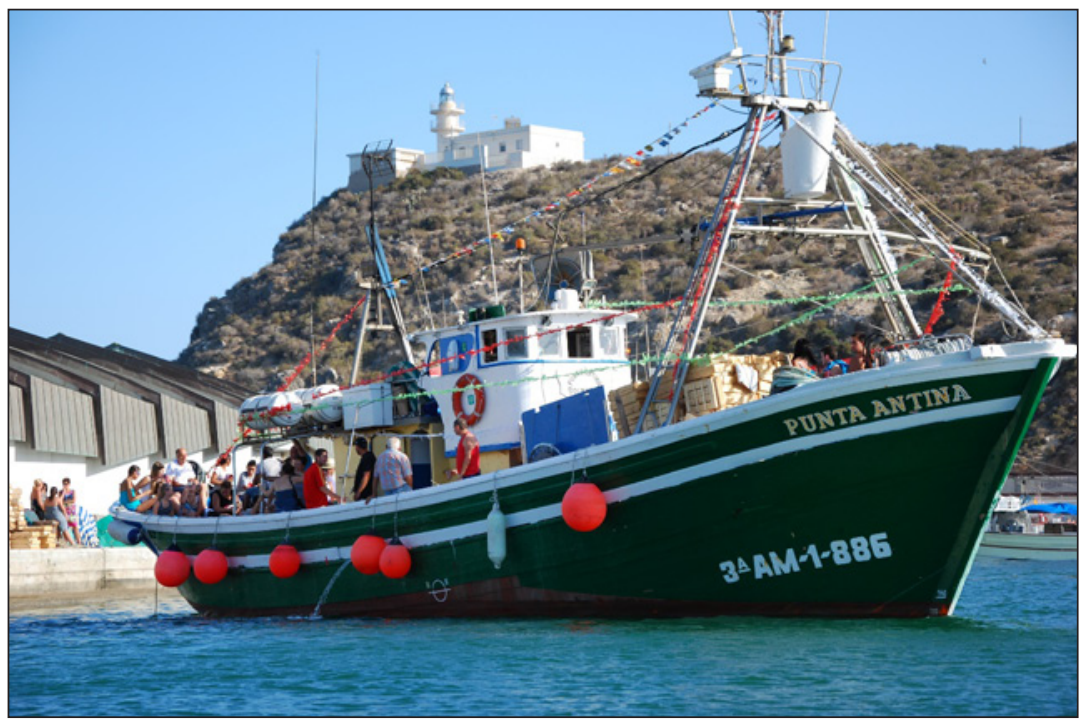

Fuente: Laura Hummer Hernández.

ción está comenzando a considerar los elementos marítimos (sobre todo los gastronómicos) como un recurso turístico de gran importancia (Rogelja y Janko, 2017). En Sicilia, los pescadores y autoridades locales han impulsado la puesta en valor de un arte de pesca tradicional en vías de desaparición, el palangre de fondo, como un atractivo turístico que genera beneficios adicionales durante el verano mediante demostraciones a bordo de las embarcaciones (Cillari et al., 2012). En lo que respecta a la Região Centro Norte de Portugal, en ella se han desarrollado varios centros de interpretación sobre la jábega, un arte de pesca de tiro que tiene una gran tradición en todo el litoral portugués (Figura 2). Además, se han restaurado las viviendas típicas de los pescadores (los palheiros), dotándolas de un uso turístico, pero sin perder su patrón arquitectónico, con el fin de que los turistas puedan recrear el modo de vida de los marineros portugueses de la primera mitad del siglo XX.

En España, el turismo marinero/pesquero ha sido desarrollado en las Comunidades Autónomas litorales, gracias al impulso de Cofradías de Pescadores, Grupos de Acción Local Pesqueros (GALP), empresas privadas y consistorios locales. Pero, interesa advertir que el éxito ha sido dispar entre todas ellas. Así, Galicia es la más prolífica en número de iniciativas, ya que se ha conseguido una alternativa de rentabilidad económica y un instrumento de puesta en valor de los numerosos recursos de las comunidades pesqueras, tanto tangibles como intangibles (Patiño, 2016). Respecto a las regiones mediterráneas, conviene mencionar el Proyecto Europeo "MARIMED: La pesca como factor de desarrollo sostenible", desarrollado entre 2004 y 2006, que tenía como fin el desarrollo de formas de turismo teniendo 


\section{Figura 2 \\ CENTRO DE INTERPRETAÇÃO DA ARTE XÁVEGA. PRAIA DA TOCHA. PORTUGAL}

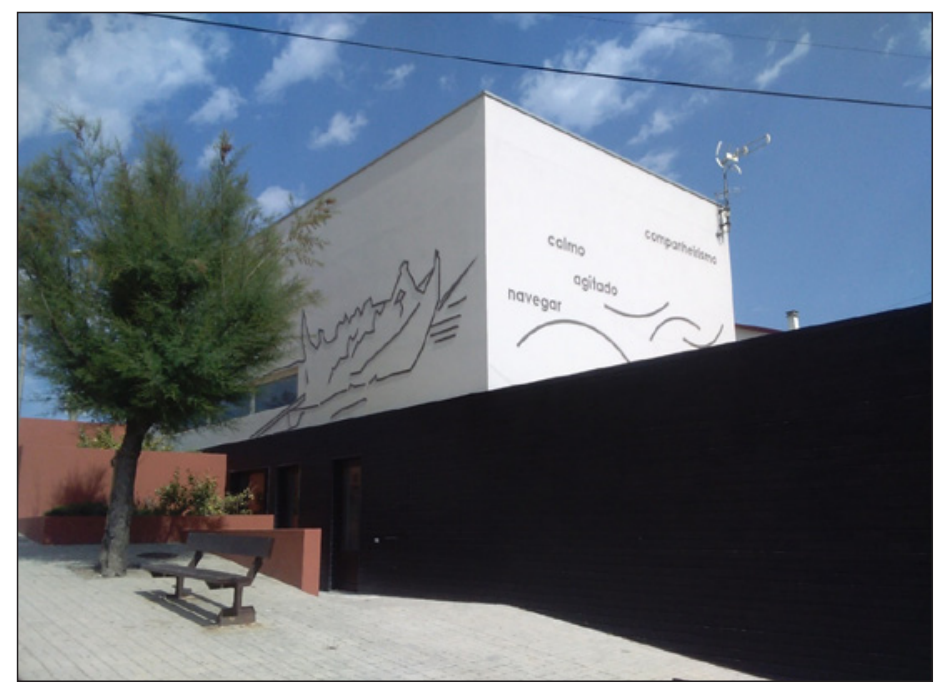

Fuente: Autor.

como elemento central e innovador la pesca, considerada como sistema social, económico y cultural, capaz de ofrecer un valor añadido al beneficio del mar (Pérez y Espejo, 2012). Esta iniciativa no tuvo un gran éxito en España. Solo algunos municipios costeros, como Águilas (Región de Murcia) o Roses (Cataluña), aprovecharon las ayudas comunitarias, creándose infraestructuras museísticas o la remodelación de los puertos pesqueros (Figura 3). No obstante, en el último lustro, el turismo marinero/pesquero se está impulsando con fuerza en la costa mediterránea española, debido a las nuevas demandas de los turistas y a la necesidad de ofrecer productos singulares de calidad que ayuden a los destinos turísticos a ser más competitivos. Por ello, se incorporan elementos relacionados con el paisaje o la identidad local, como es el caso del patrimonio vinculado a la pesca, parámetros que favorecen un turismo que aumenta la competitividad del destino, genera nuevas rentas económicas en la comunidad receptora y garantiza la sostenibilidad futura de los recursos y el territorio (Croes et al., 2018).

Una de las problemáticas que presenta el turismo marinero/pesquero en España es la diversidad de denominaciones con la que es nombrado en las distintas Comunidades Autónomas. Por ejemplo, en Galicia se denomina "turismo mariñeiro", y en Canarias "pesca-turismo" (Pardellas y Padín, 2013). Prácticamente, tan solo las leyes de pesca o/y los borradores de las que están en trámite por parte de las Comunidades Autónomas (Galicia, Cataluña, Islas Baleares, Islas Canarias y Comunidad Valenciana) son las únicas que definen claramente el concepto turismo pesquero, no existiendo una definición unánime para este término. 


\section{Figura 3 \\ CENTRO DE INTERPRETACIÓN DEL MAR. ÁGUILAS. ESPAÑA}

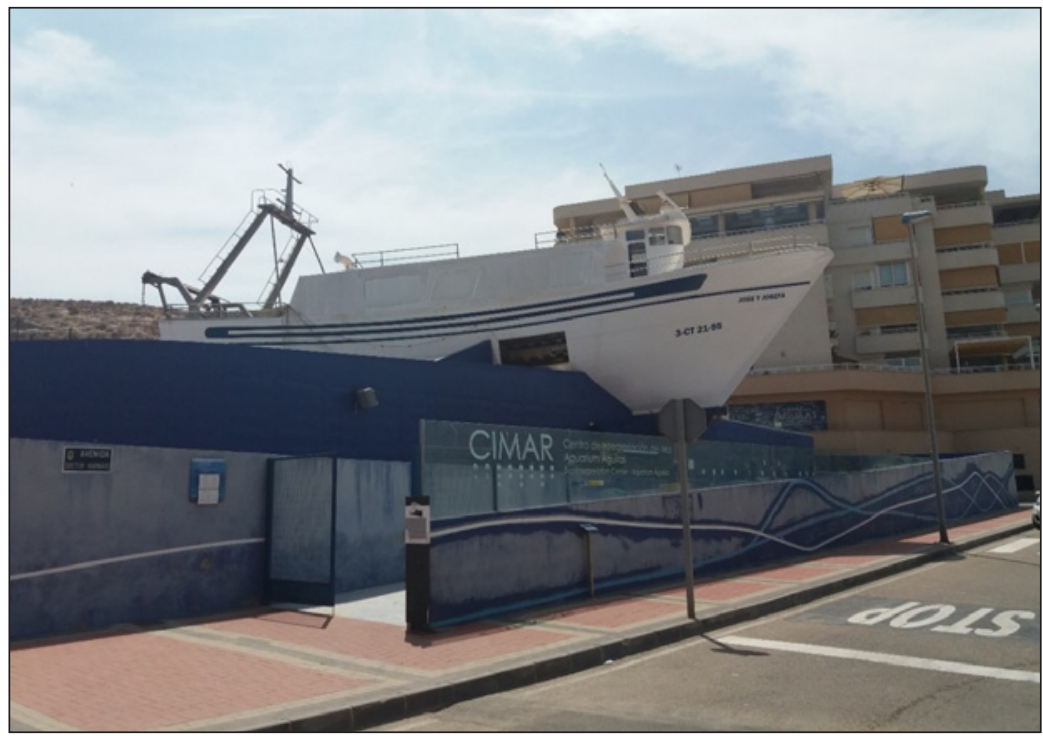

Fuente: Autor.

Por tanto, al ser una de las modalidades turísticas emergentes en los últimos años, parece oportuno abordar un trabajo de investigación en el que se realice un recorrido conceptual sobre los términos turismo marinero/pesquero y pesca-turismo. Fundamentalmente han sido abordados desde las distintas leyes de pesca, por lo que existe una carestía de definiciones desde la literatura especializada. En el presente trabajo se analizarán estas terminologías, tratando de delimitar ambas acepciones de la forma más completa posible, con vistas a la consolidación del turismo marinero/pesquero.

\section{EL TURISMO MARINERO/PESQUERO EN LA LEGISLACIÓN ESPAÑOLA}

La primera definición existente en España sobre turismo marinero/pesquero se encuentra en la Ley 11/2008, de 3 de diciembre, de Pesca de Galicia. Esta Ley, en su Artículo 112, define el turismo marinero como "Las actividades desarrolladas por los colectivos profesionales del mar, mediante contraprestación económica, orientadas a la valorización y difusión de su trabajo en el medio marino, así como de las costumbres, tradiciones, patrimonio y cultura marinera". En la actualidad, el turismo marinero en Galicia queda definido del mismo modo a través de la Ley 6/2009, de 11 de diciembre, de modificación de la Ley 11/2008, de 3 de diciembre, de Pesca de Galicia.

Un año más tarde, el Anteproyecto de Ley de Pesca Marítima, Marisqueo y Acuicultura de la Comunidad Autónoma de Canarias, de septiembre de 2009, y actualmente en debate, define en su Artículo 115 el turismo pesquero con idénticas palabras a las recogidas por la 
legislación autonómica gallega. No obstante, la definición podría variar, ya que este Anteproyecto tiene una antigüedad de 9 años, y en la actualidad (junio de 2018), se contempla su modificación para que se recojan todos los aspectos relacionados con los avances del sector pesquero y turístico.

La siguiente Comunidad Autónoma en recoger este concepto en su legislación fue Cataluña. Así, el Decreto 87/2012, de 31 de julio, sobre la pesca-turismo, el turismo pesquero y acuícola y las demostraciones de pesca en aguas marítimas y continentales de Cataluña, define en su artículo 3 el turismo pesquero y acuícola como "La actividad complementaria de la pesca profesional, el marisqueo y la acuicultura que se desarrolla fuera de embarcaciones pesqueras, en puertos pesqueros, instalaciones de acuicultura y zonas litorales o continentales relacionadas con la pesca, acuicultura y el marisqueo, por personas profesionales del sector, mediante contraprestación económicas, orientadas a la valorización y la difusión de las actividades y productos del sector así como de las costumbres, tradiciones, patrimonio y cultura marinera".

El primer autor que define el turismo marinero/pesquero ajeno a la legislación es Agustín Molina en el año 2013, con la redacción del documento Diagnóstico Estratégico de la Actividad Pesca-Turismo en España, enmarcado dentro del Proyecto SAGITAL, impulsado por la Universidad Politécnica de Madrid y desarrollado en el marco del programa EQUAL del Fondo Social Europeo. En el mismo se especifica el turismo marino o turismo pesquero como "Aquellas actividades que mediante la promoción y la difusión de la cultura pesquera tradicional, pretenden compatibilizar el mundo de la pesca profesional con la demanda turística que, especialmente en algunas épocas del año, se da en nuestras costas".

Ese mismo año, la Ley 6/2013, de 7 de noviembre, de Pesca Marítima, Marisqueo y Acuicultura en las Islas Baleares define en su Artículo 56 el turismo marinero como "Las actividades cuyo objetivo principal es diversificar la economía en las zonas pesqueras mediante el desarrollo de servicios complementarios del sector pesquero que generen puestos de trabajo, pongan de relieve los valores positivos de la actividad y contribuyan a la protección del medio ambiente y al consumo de los productos pesqueros locales". Posteriormente, a través del Decreto 22/2016, de 22 de abril, por el que se regulan las medidas para la diversificación de los sectores pesquero y acuícola en las Islas Baleares, el turismo marinero se denomina a la "Actividad que realizan los colectivos de profesionales del mar, mediante una contraprestación económica, orientada a la valoración y la difusión de las actividades y productos del medio marino, así como de las costumbres, tradiciones, cultura y patrimonio marineros, que, por ello, trasciende la mera actividad extractiva y comercial. El turismo pesquero será una actividad complementaria y simultánea a la pesca profesional'.

A nivel nacional, la Secretaría General de Pesca del Ministerio de Agricultura y Pesca, Alimentación y Medio Ambiente, elaboró un Anteproyecto de Ley, aprobado el 11 de abril de 2014, para la modificación de la vigente Ley 3/2001 de Pesca Marítima del Estado. Esta reforma introduce en el texto el concepto de turismo marinero: "Actividad desarrollada por los colectivos de profesionales del mar, mediante contraprestación económica, orientada a la valorización y difusión de las actividades y productos del medio marino, así como de las costumbres, tradiciones, patrimonio y cultura marinera". Con posterioridad, la Ley 33/2014 de 26 de diciembre, por la que se modifica la Ley 3/2001, de 26 de marzo, 
de Pesca Marítima del Estado, define el turismo marinero o pesquero como la "Actividad desarrollada por los colectivos de profesionales del mar, mediante contraprestación económica, orientada a la valorización y difusión de las actividades y productos del medio marino, así como de las costumbres, tradiciones, patrimonio y cultura marinera, que por ello trasciende la mera actividad extractiva y comercial".

Otra publicación científica, concretamente el Manual SEO/BirdLife de Buenas Prácticas Ambientales en Turismo Pesquero de 2014, que hace referencia al término, considera que éste comprende el "Conjunto de actividades que mediante la promoción y la difusión de la cultura pesquera tradicional, pretenden compatibilizar el mundo de la pesca profesional con la demanda turística. Se trata de una forma de acercar al visitante al mundo de la pesca, presentado por los propios pescadores y que puede ir desde las visitas guiadas a las lonjas, a la gestión de comercios sobre artículos marineros, museos o gastronomía".

La definición de turismo pesquero o marinero más reciente se encuentra en la Ley 5/2017, de 10 de febrero, de Pesca Marítima y Acuicultura de la Comunitat Valenciana. En ella queda definido como la "Actividad desarrollada por los colectivos de profesionales del mar, mediante contraprestación económica, orientada a la valorización y difusión de las actividades y productos del medio marino, así como de las costumbres, tradiciones, patrimonio y cultura marinera, que por ello trasciende la mera actividad extractiva y comercial".

Por tanto, tras la lectura del marco legal que le afecta en distintos ámbitos territoriales españoles, de las publicaciones especializadas y reflexionar sobre el concepto, me animo a definir el turismo marinero, que considero más acertado que turismo pesquero, como: "Las actividades desarrolladas en un entorno del litoral, a bordo o fuera de las embarcaciones pesqueras, con el fin de diversificar la economía y poner en valor una singularidad que incida positivamente en los sectores pesquero y de servicios en zonas costeras tradicionales mediante contraprestación económica, para difundir la cultura marinera, sus tradiciones, el trabajo en el medio marino y en las instalaciones portuarias, el patrimonio material e inmaterial asociado y el modo de vida de los pescadores".

\section{LA PESCA-TURISMO}

La denominada pesca-turismo es una de las actividades más singulares que se encuadran dentro del turismo marinero/pesquero, ya que se desarrolla a bordo de una embarcación, mediante la observación directa por parte del turista del trabajo de los pescadores.

La definición de este concepto se trata por primera vez en Italia, concretamente en el Decreto 293/1999 de 13 de abril de 1999, es decir, varios años antes que el término de turismo marinero/pesquero. El citado Decreto define la actividad pesca-turismo como "Aquella actividad empresarial del armador (persona física, empresa o cooperativa) de una embarcación de pesca costera local, que embarca en su propio buque personas distintas de la tripulación a fin de realizar actividades de pesca-turismo". La legislación italiana modifica con el Decreto Legislativo 226/2001, de 19 de mayo en su Artículo 3, este concepto, entendiéndose desde ese momento como "El embarque de personas que, sin formar parte de la tripulación de la nave de pesca, lo efectúan con fines turísticorecreativos y que denominamos pesca-turismo". 
El siguiente país en abordar esta definición fue Portugal, concretamente para la Región Autónoma de las Islas Azores, a través del Decreto Legislativo Regional $n^{\circ}$ 36/2008/A en su Artículo 1, el cual presenta la acepción del siguiente modo: "La Pescaturismo es la oferta de servicios marítimo turísticos de naturaleza cultural de placer, de pesca y actividades accesorias complementarias, ejercida por operador marítimo turístico con licencia en los términos de la presente norma mediante la utilización de embarcación registrada en el ejercicio de la pesca comercial".

En España, la primera definición del concepto aparece en la Ley 11/2008, de 3 de diciembre, de Pesca de Galicia. En su artículo 113, considera, dentro de las actividades que conforman el turismo marinero, la pesca-turismo como "Las actividades desarrolladas a bordo de embarcaciones pesqueras por parte de los profesionales del mar dirigidas al conocimiento, valorización y difusión de su trabajo en el medio marino". Tomando como ejemplo la definición de la legislación gallega, el Anteproyecto de Ley de Pesca Marítima, Marisqueo y Acuicultura de Canarias, de septiembre de 2009, define la actividad pesca-turismo del mismo modo que la Ley 11/2008, de 3 de diciembre, de Pesca de Galicia. Pero, tal y como se advierte para el concepto de turismo marinero/ pesquero, este podría modificarse debido a la necesidad de adaptarse a las nuevas definiciones.

Padín y Aboy (2010), definen por primera vez en la literatura científica española el concepto de pesca-turismo. Sin embargo, se trata de una definición somera. Los citados autores entienden el término como "La ocupación vinculada al sector pesquero, que desarrolla una actividad lúdica y de divulgación, dando a conocer la profesión a bordo del buque de pesca".

Dos años más tarde, el Decreto 87/2012, de 31 de julio, sobre la Pesca-Turismo, el Turismo Pesquero y Acuícola y las Demostraciones de Pesca en Aguas Marítimas y Continentales de Cataluña, denomina la actividad pesca-turismo como "La actividad complementaria de la pesca profesional consistente en la pesca extractiva o de demostración a bordo de embarcaciones pesqueras por personas profesionales del sector, mediante contraprestación económica, orientadas a la valorización y la difusión de las actividades y productos del sector así como de las costumbres, tradiciones, patrimonio $y$ cultura marinera.

Seguidamente, Molina (2013), en el citado Diagnóstico Estratégico de la Actividad Pesca-Turismo en España, acuña el término como "Aquellas actividades realizadas por los profesionales de la pesca marítima que, teniendo por finalidad la difusión, la valorización y la promoción de los modos de vida, las costumbres y la cultura de las personas dedicadas a la pesca marítima, posibilitan que terceros ajenos a la tripulación puedan, mediante contraprestación económica, embarcar en los buques de pesca con el fin de observar y conocer las faenas de pesca, los instrumentos de navegación, las rutas del litoral o el medio marino".

En el Artículo 56 de la Ley 6/2013, de 7 de noviembre, de Pesca Marítima, Marisqueo y Acuicultura en las Islas Baleares, se entiende la pesca-turismo como "La actividad de turismo marinero desarrollada a bordo de embarcaciones por profesionales del sector con personas distintas a la tripulación, mediante una contraprestación económica, que tiene por objeto, directa o indirectamente, la difusión, la valorización y la promoción del 
modo de vida, de las costumbres y de la cultura de la actividad pesquera". No obstante, este término se modifica tres años más tarde, concretamente con el Decreto 22/2016, de 22 de abril, por el que se regulan las medidas para la diversificación de los sectores pesquero y acuícola en las Islas Baleares, la actividad Pesca-Turismo queda definida como "La modalidad de turismo pesquero o marinero que realizan profesionales de este sector a bordo de embarcaciones pesqueras, mediante una contraprestación económica, que tiene por objeto, directa o indirectamente, la difusión, valoración y promoción del modo de vida, costumbres y cultura de la actividad pesquera. En cualquier caso, la actividad pesquera solo puede ser ejercida por los miembros de la tripulación de la embarcación".

A nivel nacional, la Ley 33/2014 de 26 de diciembre, por la que se modifica la Ley 3/2001, de 26 de marzo, de Pesca Marítima del Estado, reconoce la modalidad pescaturismo como "El tipo de actividad de turismo pesquero o marinero desarrollada a bordo de embarcaciones pesqueras por parte de profesionales del sector, mediante contraprestación económica, que tiene por objeto la valorización y difusión de su trabajo en el medio marino, en la que los turistas embarcados no podrán ejercer la actividad pesquera".

También en 2014, el Manual SEO/BirdLife de Buenas Prácticas Ambientales en Turismo Pesquero propone la modalidad pesca-turismo como "La actividad de turismo pesquero consistente en acompañar a la tripulación de un barco durante una jornada de pesca, aprendiendo directamente de los profesionales a valorar el medio marino y sus recursos. Embarcados en los barcos de pesca, los turistas observan y conocen las faenas de pesca, los instrumentos de navegación, las rutas o el medio marino".

Tras analizar las definiciones existentes y estudiar la actividad pesca-turismo, considero que ésta puede ser definida como "La actividad insertada dentro del marco del turismo marinero/pesquero, desarrollada a bordo de embarcaciones pesqueras, consistente en acompañar a la tripulación de un barco durante una jornada completa de pesca, dirigida por profesionales del sector mediante contraprestación económica, con el fin de poner en valor y difundir su trabajo en el medio marino, permitiendo al turista conocer el sistema de pesca, funcionamiento de radares, fases del calado del arte y la clasificación del pescado".

\section{REFLEXIONES FINALES}

Esta modalidad turística lleva una corta trayectoria, algo más de una década, en todas las escalas. Sin embargo, en un contexto de crisis del sector pesquero y las nuevas motivaciones de los turistas, que buscan experiencias originales en una combinación de vacaciones, turismo y enriquecimiento cultural (García y Albuquerque, 2003), se convierte en un objetivo ineludible para el desarrollo territorial e identitario de los territorios costeros con tradición pesquera.

En España, el turismo marinero/pesquero presenta una tendencia al alza, así queda constatado mediante el creciente número de iniciativas. Sin embargo, no todas las Comunidades Autónomas litorales cuentan con una legislación que ayude al impulso de estas actividades, especialmente la pesca-turismo. Por ejemplo, este hecho está frenando su 
implantación en lugares como la Región de Murcia o las Islas Canarias, cuyos pescadores en la actualidad no pueden llevar en sus embarcaciones a turistas para que contemplen el desarrollo de una jornada completa de pesca (Figura 4).

En definitiva, el tiempo dirá si el turismo marinero/pesquero se consolida dentro de la oferta turística de las localidades vinculadas a la pesca existentes, no solo en España, sino también en otros lugares del mundo. La cooperación y predisposición por parte de los actores implicados (pescadores, administración pública, cofradías de pescadores y empresas privadas) será decisiva a la hora de promover esta modalidad.

Figura 4

PESCADORES FAENANDO EN LA BAHÍA DE MAZARRÓN. ESPAÑA

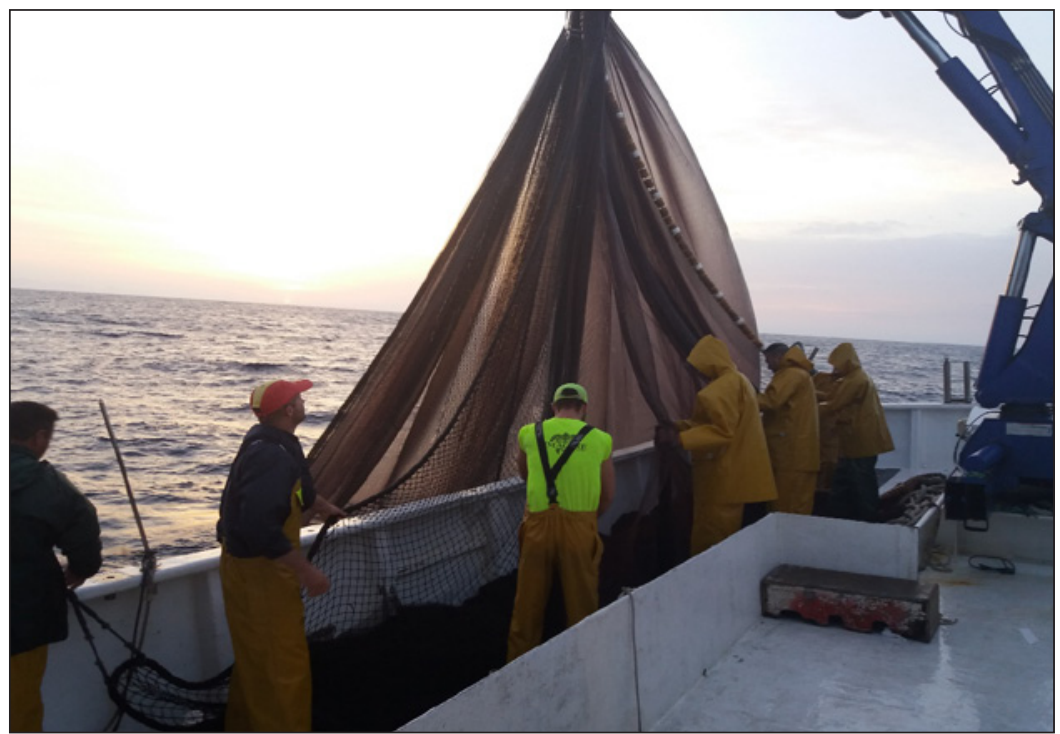

Fuente: Autor.

\section{BIBLIOGRAFÍA}

CAPEL SÁEZ, H. (2014): El patrimonio: la construcción del pasado y del futuro. Barcelona, Ediciones del Serbal.

CHEN, C.L. y CHANG, Y.C. (2017): «A transition beyond traditional fisheries: Taiwan's experience with developing fishing tourism». Marine Policy, vol. 79, 84-91.

CHEONG, S. M. (2003): «Privatizing tendencies: fishing communities and tourism in Korea». Marine Policy, vol. 27 (1), pp. 23-29.

Cillari, T., FAlAutANO, M., CASTRIOTA, L., MARINO, V., VIVONA, P. y ANDALORO, F. (2012): «The use of bottom longline on soft bottoms: An opportunity of development for fishing tourism along a coastal area of the Strait of Sicily (Mediterranean Sea)». Ocean \& Coastal Management, vol. 55, pp. 20-26. 
CROES, R., RIDDERSTAAT, J. y VAN NIEKERK, M. (2018): «Connecting quality of life, tourism specialization, and economic growth in small island destinations: The case of Malta». Tourism Management, vol. 65, pp. 212-223.

DECRETO 87/2012, de 31 de julio, sobre la pesca-turismo, el turismo pesquero y acuícola y las demostraciones de pesca en aguas marítimas y continentales de Cataluña. Diari Oficial de la Generalitat de Catalunya, nº.184, de 2 de agosto de 2012.

DECRETO 22/2016, de 22 de abril, por el que se regulan las medidas para la diversificación de los sectores pesquero y acuícola en las Islas Baleares. Boletín Oficial de las Islas Baleares, $\mathrm{n}^{\circ} 51$, de 23 de abril de 2016.

DECRETO LEGISLATIVO REGIONAL No. 36/2008/A de 30 de Julho, que Define o quadro legal da pesca-turismo exercida nas águas da subárea dos Açores da zona económica exclusiva (ZEE) portuguesa, Jornal Oficial Presidência do Governo dos Açores. $\mathrm{n}^{\circ} 149$, do 9 de agosto de 2008.

DECRETO MINISTERIALE 293/1999 de 13 de abril de 1999, Regolamento recante norme in materia di disciplina dell'attivita' di pescaturismo, in attuazione dell'art. 27-bis della legge 17 febbraio 1982, n. 41, e successive modificazioni. Gazzetta Ufficiale della Repubblica Italiana, n ${ }^{\circ}$ 197, di 23 de agosto di 1999.

GARCÍA SÁNCHEZ, A. y ALBUQUERQUE GARCÍA, F.J. (2003): «El turismo cultural y el de sol y playa ¿Sustitutivos o complementarios?». Cuadernos de Turismo, $\mathrm{n}^{\circ} 11$, pp. 97-105.

HERRERA-RACIONERO, P., MIRET-PASTOR, L. y LIZCANO, E. (2018): «Viajar con la tradición: los pescadores artesanales ante la pesca-turismo en la Comunidad Valenciana (España)». Cuadernos de Turismo, no 41, pp. 279-293.

LEY 11/2008, de 3 de diciembre, de Pesca de Galicia. Boletín Oficial del Estado (BOE), $\mathrm{n}^{\mathrm{o}} 15$, de 17 de enero de 2009.

LEY 6/2009, de 11 de diciembre, de modificación de la Ley 11/2008, de 3 de diciembre, de Pesca de Galicia. Boletín Oficial del Estado (BOE), $\mathrm{n}^{\circ} 30$, de 4 de febrero de 2010.

LEY 6/2013, de 7 de noviembre, de pesca marítima, marisqueo y acuicultura en las Islas Baleares, Boletín Oficial del Estado (BOE), n 290, de 4 de diciembre de 2013.

LEY 33/2014 de 26 de diciembre, por la que se modifica la Ley 3/2001, de 26 de marzo, de Pesca Marítima del Estado. Boletín Oficial del Estado (BOE), n ${ }^{\circ} 313$, de 27 de diciembre de 2014.

LEY 5/2017, de 10 de febrero, de pesca marítima y acuicultura de la Comunitat Valenciana. Boletín Oficial del Estado (BOE), n 56, de 7 de marzo de 2017.

MARTÍNEZ HERNÁNDEZ, C. y MORENO MUÑOZ, D. (2013): «La pesca como recurso turístico en Mazarrón (Murcia)». Estudios Turísticos, no 196, pp. 113-131.

MOLINA GARCÍA, A. (2013): Diagnóstico estratégico de la actividad Pesca-turismo en España. Madrid: Universidad Politécnica de Madrid y Ministerio de Agricultura, Alimentación y Medio Ambiente.

MORENO MUÑOZ, D. (2016): «Turismo marinero ¿Complemento del modelo turístico de sol y playa en la Región de Murcia?». En X.M. Santos, P. Taboada y L. López (Eds.) Actas del X Congreso Internacional de Turismo Rural y Desarrollo Sostenible Santiago de Compostela, España: Universidad de Santiago de Compostela, pp. 411-420. 
MORENO MUÑOZ, D. (2018): La actividad pesquera en la Bahía de Mazarrón y el turismo marinero como propuesta de desarrollo (Tesis Doctoral). Universidad de Murcia, España.

PADIN FABEIRO, C. y ABOY GARCÍA, S. (2010): «Nuevos productos turísticos: la apuesta por el turismo marinero en Galicia». Tékhne-Revista de Estudos Politécnicos, vol. 14, pp. 55-83.

PARDELLAS DE BLAS, X.X. y ESPEJO MARÍN, C. (2013): «Turismo pesquero». En E.A. Del Valle Tuerro y D. López Olivares (Eds.) La actividad turística española en 2012. Madrid, Editorial Universitaria Ramón Areces, pp. 41-48.

PARDELLAS DE BLAS, X.X. y PADÍN FABEIRO, C. (2013): «La nueva demanda combinada de turismo litoral y turismo pesquero: motivaciones y efectos», Cuadernos de Turismo, $\mathrm{n}^{\mathrm{O}} 32$, pp. 247-262.

PATIÑO ROMARÍS, C.A. (2016): «El turismo marinero: un producto diferenciador y emergente de la oferta turística del litoral gallego». En X.M. Santos, P. Taboada y L. López (Eds.), Actas del X Congreso Internacional de Turismo Rural y Desarrollo Sostenible. Santiago de Compostela, España: Universidad de Santiago de Compostela, pp. 401-410.

PÉREZ PIERNAS. P. y ESPEJO MARÍN, C. (2012): «La pesca como factor de desarrollo del turismo sostenible. El caso de Águilas (Murcia)». Cuadernos de Turismo, $\mathrm{n}^{\circ} 30$, pp. 267-284.

ROGELJA, N. y JANKO, A. (2017): «How authentic is fishing tourism in Slovenia?» En N. Rogelja y A. Janko (Eds.) Fish on the Move. Cham, Suiza: Springer International Publishing, pp. 145-170.

SEO/BIRDLIFE (2014): Manual SEO/BirdLife de Buenas Prácticas Ambientales en Turismo Pesquero. Madrid, España: Sociedad Española de Ornitología.

VASCONCELOS, V., DE SOUSA, R., y SANTOS, A. (2014): «Artesanato local e atividade pesqueira na comunidade do Carnaubal (Luís Correira, Piauí-Brasil) como fatores para o desenvolvimento sustentável do turismo». TURyDES, Vol. 7 (16). 